\title{
Riesgos Psicosociales Laborales, Características Organizacionales y Salud Mental: El Rol Mediador de la Violencia Laboral
}

\section{Occupational Psychosocial Risks, Organizational Characteristics, and Mental Health: The Mediating Role of Workplace Violence}

\author{
Andrea Palma ${ }^{1,2}$, Mónica M. Gerber ${ }^{1}$ y Elisa Ansoleaga ${ }^{1}$ \\ ${ }^{1}$ Facultad de Psicología, Universidad Diego Portales \\ 2 Facultad de Psicología, Universidad San Sebastián
}

\begin{abstract}
La exposición a riesgos psicosociales y violencia laboral se asocia con una mayor presencia de problemas de salud mental en los/as trabajadores/as. El presente estudio tuvo como objetivo indagar el rol mediador de la violencia laboral entre los riesgos psicosociales (ISOSTRAIN, desbalance esfuerzo-recompensa), antecedentes organizacionales (liderazgo tiránico) y problemas de salud mental (distrés) en 1023 trabajadores/as de tres hospitales públicos chilenos de alta complejidad de tres regiones del país. Se hipotetizó que la exposición a riesgos psicosociales y algunas características organizacionales (liderazgo tiránico) se asocia a menores niveles de salud mental y que parte de este efecto se encuentra mediado por la exposición a violencia laboral. Se aplicó un cuestionario incluyendo el Negative Act Questionnaire Revised (NAQ-R), el Screening de Distrés Psicológico, la Escala de Liderazgo Destructivo, el Cuestionario de Desbalance Esfuerzo-Recompensa y el Job Content Questionnaire. Mediante análisis de senderos, se observó que la exposición a riesgos psicosociales, liderazgo tiránico y violencia laboral aumenta los niveles de distrés de los/as trabajadores/as. Parte del efecto de los riesgos psicosociales y el liderazgo tiránico en la salud mental se encuentra mediado por la exposición a violencia laboral. El artículo termina discutiendo la necesidad de avanzar en protocolos y prevención de riesgos psicosociales y violencia laboral, en particular en el contexto del trabajo en salud pública.
\end{abstract}

Palabras clave: violencia laboral, riesgos psicosociales, salud mental, distrés, liderazgo

\begin{abstract}
Exposure to psychosocial risks and workplace violence is associated with a higher prevalence of workers' mental health problems. The purpose of the present study was to examine the mediating role of workplace violence between psychosocial risks (ISOSTRAIN, effort-reward imbalance), organizational background (tyrannical leadership) and mental health problems (distress) among 1023 workers in three high complexity public hospitals in three regions of Chile. It was hypothesized that exposure to psychosocial risks and some organizational features (tyrannical leadership) is associated with lower levels of mental health and that part of this effect is mediated by exposure to workplace violence. A questionnaire was applied including the Negative Act Questionnaire Revised (NAQ-R), the Psychological Distress Screening, the Destructive Leadership Scale, the Effort-Reward Imbalance Questionnaire and the Job Content Questionnaire. Path analysis showed that exposure to psychosocial risks, tyrannical leadership and workplace violence increase levels of workers' distress. Part of the effect of psychosocial risks and tyrannical leadership on mental health is mediated by exposure to workplace violence. The article finishes by discussing the need to move forward in developing protocols and prevention of psychosocial risks and workplace violence, particularly in the context of public health work.
\end{abstract}

Keywords: workplace violence, psychosocial risks, mental health, distress, leadership

El trabajo constituye un elemento central en la vida de las personas, pudiendo promover su desarrollo y potenciar sus capacidades, mientras que también puede atrofiar sus capacidades cuando las condiciones

Andrea Palma iD https://orcid.org/0000-0002-6281-1509

Mónica M. Gerber iD https://orcid.org/0000-0003-2589-199X

Elisa Ansoleaga (iD https://orcid.org/0000-0003-0182-0126

Este artículo fue realizado en el marco del Proyecto FONDECYT Regular $2017 \mathrm{~N}^{\circ} 1170239$, "Prevalencia, formas de manifestación y factores facilitadores de la violencia laboral y sus efectos en la salud mental en población asalariada en Chile, atendiendo desigualdades sociales y de género", el cual cuenta con financiamiento de la Comisión Nacional de Investigación Científica y Tecnológica de Chile (CONICYT) y la Beca CONICYT de doctorado nacional $N^{\circ}$ 21170967. Existe un primer artículo ya publicado basado en esta investigación: Palma-Contreras \& Ansoleaga (2020), en el que se describen las asociaciones entre factores de riesgos psicosociales, dimensiones organizacionales y problemas de salud mental, relacionados con la violencia laboral.

La correspondencia relativa a este artículo debe ser dirigida a Andrea Palma, Facultad de Psicología, Universidad San Sebastián, Lota 2465, Providencia, Santiago, Región Metropolitana, Chile. Email: andrea.m.palma.c@gmail.com 
laborales y organizacionales no son las adecuadas (Hopenhayn, 2001). El objetivo de este artículo fue indagar en el rol que juegan los riesgos psicosociales como antecedentes de dos posibles consecuencias negativas en el contexto laboral: la violencia laboral (entendida como intimidación en el lugar de trabajo) y los problemas de salud mental (particularmente, el nivel de distrés; en adelante VL).

Considerando los cambios que ha sufrido la organización del trabajo en las últimas décadas (polifuncionalidad, flexibilidad laboral, precarización del empleo, entre otros; Stecher Guzmán, 2012) y, en particular, los cambios vividos en el sector público, se examina el rol de la tensión laboral, el bajo apoyo social, el desbalance entre esfuerzo y recompensa, así como la exposición a liderazgos tiránicos en la experimentación de VL y en problemas de salud mental en trabajadores/as de tres hospitales públicos en Chile. Un considerable número de estudios han encontrado que la exposición a riesgos psicosociales se asocia con una mayor experimentación de VL (e.g., Feijó et al., 2019; Rodwell \& Demir, 2012; Wei et al., 2016) y problemas de salud mental (e.g., Karasek \& Theorell, 1990; Siegrist et al., 2009). El presente estudio pretendió extender esta investigación, evaluando un modelo que hipotetiza que parte de la razón por la que los riesgos psicosociales inciden negativamente en la salud mental de los/as trabajadores/as se debe a que ponen a estos en mayor riesgo de experimentar VL. De esta forma, a diferencia de estudios previos, se propone que la VL podría cumplir un rol mediador entre los riesgos psicosociales y el nivel de distrés.

Entender los factores que se encuentran a la base de la exposición a VL y el nivel de distrés de los trabajadores/as de salud resulta fundamental por al menos tres razones. Primero, porque los problemas de salud mental representan una de las principales causas de enfermedad mundial y ausentismo laboral (Mnookin, 2016; Tsutsumi et al., 2015), encontrándose los funcionarios de salud entre los grupos de alto riesgo de ser víctimas del fenómeno (Organización Internacional del Trabajo [OIT] et al., 2002). Segundo, porque los funcionarios de salud proveen un servicio de primera necesidad para la población, mientras que la calidad de la atención depende de la situación de los funcionarios del área (World Health Organization [WHO], 2000). Así, la exposición de funcionarios del ámbito de la salud a VL repercute negativamente en la atención de los usuarios (Departamento de Salud Ocupacional, 2013). Tercero, porque si bien entender la salud mental y la exposición a la VL en el ámbito de la salud resulta de suma urgencia, tal como indicó la revisión sistemática de la literatura realizada por Toro y Gómez-Rubio (2016) en Chile, solo existen estudios exploratorios y descriptivos realizados en poblaciones específicas en relación al tema, abriendo la necesidad de avanzar en el análisis de la salud mental y la VL en ámbitos nuevos, así como en el entendimiento de los factores que se encuentran a la base de este fenómeno.

Este artículo está estructurado de la siguiente manera. Primero, se analiza el mundo del trabajo en Chile y los cambios sufridos en la organización del mismo en las últimas décadas. Se pone un especial énfasis en el caso del trabajo en el sector público. Segundo, se describen los conceptos de VL y distrés, dando cuenta de los antecedentes que la literatura ha reconocido como relevantes. Tercero, en función de los antecedentes, se propone un modelo que vincula los distintos conceptos y se especifican las hipótesis de trabajo. Cuarto, se presenta la metodología de la investigación, para concluir con resultados y reflexiones finales.

\section{Características de la Organización del Trabajo en Salud}

El trabajo en el área de la salud en Chile ha enfrentado un conjunto de cambios, de la mano de transformaciones globales en el mundo del trabajo. Los modelos de modernización de las grandes empresas introdujeron, desde la década de los 70, cambios en la organización de la manera de trabajar. Estos cambios incluyen la autoactivación, polivalencia y polifuncionalidad, mecanismos de mejora continua, sometimiento a políticas de flexibilidad laboral y a la "creciente centralidad simbólica y práctica que adquiere la figura del cliente como principio organizador y activador del proceso de trabajo" (Stecher Guzmán, 2012, p. 82). Ello ha traído consecuencias, como la precarización de los empleos, la desprotección de los/as trabajadores/as y la intensificación del trabajo, entre otras (Araujo, 2014).

El sector público no ha estado ajeno a estos cambios: la gestión pública de las últimas tres décadas en Chile se ha caracterizado por un foco en la rendición de cuentas, la flexibilización contractual y la privatización de los servicios (Fardella et al., 2016). Las transformaciones de los modelos de gestión y organización del trabajo en el área de salud han sido consecuencia del proceso de modernización del Estado que se inició en la década del 80 del siglo pasado y que se intensificó a partir del año 2002 con el último proceso de reforma sanitaria que se sustentaba en valores de equidad, solidaridad, eficiencia en el uso de los recursos y participación social (Artaza, 2012). Este proceso de modernización tiene como base el paradigma del new public management, el cual corresponde a un "enfoque administrativo que instala a la empresa 
privada como el principal modelo de eficiencia y eficacia a imitar por los servicios públicos" (Sisto, 2012, p. 36). Según Ansoleaga Moreno et al. (2012), la instalación de este enfoque en el sistema de salud se ha traducido en cambios importantes en la organización del trabajo. Así, se ha afectado el modelo de atención, la gestión de recursos humanos, los sistemas de control, el uso de indicadores, los procesos de evaluación de desempeño —que se vinculan al establecimiento de metas individuales o colectivas- y cambios en la asignación de recompensas.

\section{Riesgos Psicosociales, Violencia Laboral y Salud Mental}

El estudio de la VL ha transitado desde modelos que ponen el foco en las características personales de víctima y victimario hacia modelos que ponen el énfasis en las interacciones entre ambos para, finalmente, llegar hasta los modelos que consideran características del ambiente laboral y las culturas organizacionales, así como también un marco socio ocupacional más amplio (Einarsen, 2000). Este trabajo se inscribe en el último modelo.

En este artículo se examina la relación entre riesgos psicosociales, VL y salud mental en trabajadores/as del ámbito de salud pública en Chile. Los importantes cambios en la organización del trabajo en salud han abierto el cuestionamiento sobre posibles factores de riesgo psicosociales. Los riesgos psicosociales son "aquellas características de las condiciones de trabajo y, sobre todo, de su organización que afectan a la salud de las personas a través de mecanismos psicológicos y fisiológicos" (Moncada et al., 2002, p. 14). Entre los riesgos psicosociales se encuentran: altas exigencias psicológicas, escasas posibilidades de trabajo activo (influencia y control sobre el trabajo), bajo apoyo social e insatisfacción con las compensaciones (Candia et al., 2016).

A su vez, la VL ha sido considerada en este trabajo desde el concepto de intimidación en el lugar de trabajo (Notelaers \& Einarsen, 2013), esto es, "la exposición directa a un comportamiento negativo, sistemático y prolongado en el trabajo" (p. 671) proveniente de superiores, compañeros de trabajo, subalternos y/o usuarios. Atendiendo a la centralidad del trabajo ya mencionada, la VL ha sido considerada la epidemia del siglo XXI, siendo identificada como uno de los mayores estresores en el trabajo, debido a su magnitud y a los nocivos efectos en las personas y las organizaciones (Di Martino, 2002; Di Martino et al., 2003). Sin embargo, la evidencia disponible en Chile resulta insuficiente para ilustrar la magnitud y el impacto del fenómeno. Particularmente, el trabajo en el sector salud ha sido identificado como uno de los con mayor riesgo de exposición a VL (OIT et al., 2002). Tal es el impacto de la VL en el sector salud que se evidencian repercusiones negativas en la atención a pacientes (Cavanaugh et al., 2014; Yada et al., 2014), puesto que la calidad de atención se encuentra mediada por los funcionarios del área (WHO, 2000).

Por último, la salud mental es definida como la ausencia de sintomatología de problemas de salud mental (Lopera, 2014). En esta investigación fue operacionalizada por medio del constructo de distrés, entendido como el malestar psicológico compuesto por síntomas de depresión, inquietud, fatiga, sentimiento de culpa y ansiedad (Kessler et al., 2002).

Ahora bien, ¿cómo se relacionan los riesgos psicosociales, la exposición a VL y la salud mental de los/as trabajadores/as?

Primero, la evidencia internacional muestra que tanto la exposición a riesgos psicosociales como a VL se vinculan con peores indicadores de salud mental (Einarsen \& Nielsen, 2015; Magnavita, 2014). Dos modelos teóricos son particularmente relevantes para entender la vinculación entre riesgos psicosociales y salud mental.

El modelo de Karasek y Theorell (1990) plantea que los/as trabajadores/as que poseen altas demandas psicológicas y escasa posibilidad de tomar decisiones respecto del proceso de trabajo y el uso de habilidades perciben mayores niveles de tensión laboral (jobstrain) y que ello repercute en su salud física y mental. Johnson (1986, en Karasek et al., 1998) agregó que el apoyo social —entendido como el apoyo instrumental y socioemocional de parte de compañeros de trabajo y supervisores- modera los efectos de la presión laboral en la salud. Así, representa el tercer componente del modelo de Karasek et al. (1998), puesto que, si se agrega a la tensión psíquica, el riesgo de enfermar aumenta.

El modelo de Siegrist et al. (2009) señala que la existencia de un desbalance entre esfuerzo-recompensa aumenta la susceptibilidad de presentar estrés laboral y enfermedades.

Estudios empíricos indican que variables de los dos modelos mencionados están relacionadas con la salud mental (Cheng \& Cheng, 2017; Niedhammer et al., 2016; Russo et al., 2019; Salas et al., 2015). Por ejemplo, la latitud decisional, el apoyo social y las recompensas contribuyen a explicar las diferencias en 
sintomatología depresiva (Niedhammer et al., 2016); las tareas repetitivas, el bajo apoyo social y la poca autonomía se asocian con problemas de salud mental (Russo et al., 2019); mayores demandas psicológicas e inseguridad laboral se asocian con trastornos mentales menores (Cheng \& Cheng, 2017) y la alta tensión laboral aumenta la probabilidad de angustia (Salas et al., 2015). Adicionalmente, se ha encontrado que las largas horas en el trabajo se relacionan con sintomatología de salud mental (Gong et al., 2014) y que las mejoras en el entorno de trabajo pueden reducir los resultados negativos en la salud (Aronsson et al., 2019). En Chile, también se ha encontrado que los funcionarios públicos expuestos a riesgo psicosocial presentan mayor chance de exhibir problemas de salud mental (Ansoleaga, 2015).

A su vez, la evidencia también sugiere que la VL es un predictor relevante para entender la salud mental de las personas (Baby et al., 2014, Dement et al., 2014; Einarsen \& Nielsen, 2015; Itzhaki et al., 2015; Magnavita, 2014; Pien et al., 2015; Rodwell \& Demir, 2012; Zhao et al., 2018). Estar expuesto a VL se relaciona con problemas de salud mental, como trastorno de estrés post traumático (Baby et al., 2014), uso de fármacos antidepresivos y ansiolíticos (Dement et al., 2014) y estrés laboral (Itzhaki et al., 2015; Magnavita, 2014), entre otros.

Por último, ¿cuál es la relación entre riesgos psicosociales y VL?

De manera consistente con los modelos antes referidos, la exposición a riesgos psicosociales en el trabajo (ISOSTRAIN dado por la combinación de altas demandas psicológicas, baja latitud decisional y bajo apoyo social; desbalance esfuerzo-recompensa), sumada a distintas características organizacionales (liderazgo tiránico), se ha vinculado también a una mayor exposición a situaciones de VL (Arnetz et al., 2018; Aronsson et al., 2019; Fontes et al., 2019; Francioli et al., 2018; Olsen et al., 2017). Estudios han evidenciado que la experimentación de VL se relaciona con distintos indicadores de riesgos psicosociales: las demandas del trabajo y los recursos (control sobre el trabajo, apoyo social, recompensas salariales y posibilidades de ascenso; Balducci et al., 2011; Rodwell \& Demir, 2012); la tensión laboral (Feijó et al., 2019), particularmente cuando se encuentra asociada a bajo apoyo social (ISOSTRAIN; Arnetz et al., 2018); el desequilibrio esfuerzorecompensa (Feijó et al., 2019); la realización de tareas monótonas, la baja satisfacción con las condiciones de trabajo, la falta de percepción de oportunidades de promoción (Ariza-Montes et al., 2013) y la sobrecarga de trabajo en el área de salud (Wei et al., 2016). A su vez, entre los factores que son protectores ante la VL se encuentra la utilización de habilidades (Notelaers et al., 2010), los recursos laborales, como el desarrollo y el apoyo de colegas (Olsen et al., 2017), y el apoyo social (Attell et al., 2017; Baby et al., 2014).

Como se mencionó anteriormente, del conjunto de dimensiones de riesgos psicosociales laborales, existen dos modelos que han sido ampliamente testeados a nivel mundial respecto de sus nocivos efectos en la salud mental. De una parte, el modelo de Karasek et al. (1998) incluye las variables de demandas psicológicas, latitud decisional y soporte social. El modelo plantea que los trabajadores experimentan alta tensión psíquica (jobstrain) cuando están expuestos a altas demandas psicológicas y escasa latitud decisional. Si a ello se agrega un bajo soporte social (dando lugar al concepto de ISOSTRAIN) se aumenta el riesgo de sintomatología física y psicológica (Ansoleaga Moreno, 2013). De otra parte, está el modelo de Siegrist et al. (2009), que plantea el concepto de desbalance esfuerzo-recompensa, que se produce cuando un trabajador de forma sostenida en el tiempo enjuicia que el esfuerzo realizado en su trabajo supera las recompensas que obtiene, lo que amplía la probabilidad de presentar estrés.

Cabe señalar que Ansoleaga et al. (2019) establecen que el ISOSTRAIN es una variable de control relevante en la relación entre vulnerabilidad laboral y VL.

De acuerdo a la evidencia presentada, es posible argumentar que los riesgos psicosociales laborales serán antecedentes relevantes para explicar la salud mental de los/as trabajadores/as, así como la exposición a VL.

\section{Factores Organizacionales, Violencia Laboral y Salud Mental}

Para entender la VL y la salud mental de trabajadores/as resulta también pertinente atender a factores organizacionales. La literatura ha evidenciado que los estilos de liderazgo presentan un fuerte vínculo con la VL y la salud mental. Tal es el vínculo entre el liderazgo y la VL, que Ashforth (1994) definió un tipo de VL interna (mobbing) como la tiranía de un líder que muestra su poder por medio de la arbitrariedad, el auto engrandecimiento y el menosprecio de los subordinados. Particularmente, la exposición a liderazgo tiránico - en que el líder manipula y humilla a los subordinados para que hagan el trabajo con el fin de resguardar los intereses de la organización, sin importar el bienestar de los/as trabajadores/as (Einarsen et al., 2007, 2010) — se ha relacionado positivamente con la VL (Hoel et al., 2010). 
De acuerdo con lo anterior, es posible señalar que la calidad del liderazgo desempeña un papel central en el establecimiento de condiciones de trabajo que pueden conducir o prevenir experiencias de VL (Francioli et al., 2018).

A su vez, la exposición a determinados estilos de liderazgo puede influir positiva o negativamente en la salud mental de los/as trabajadores/as. Así, Stordeur et al. (2001) indican que el liderazgo influye en el agotamiento emocional de las enfermeras. A su vez, Nielsen et al. (2005, 2013) han reportado que el liderazgo laissez faire y la exposición a acoso laboral son los predictores más robustos del distrés posterior y que los comportamientos de liderazgo tiránico constituyen un factor de estrés severo, aun después de controlar por los efectos de los comportamientos de intimidación. Esto último se condice con la relación hipotetizada en esta investigación entre liderazgo y distrés. Por otra parte, Spence Laschinger et al. (2012) mostraron que en enfermeras canadienses recién graduadas el liderazgo auténtico se relacionaba de manera indirecta con el agotamiento emocional a través de la intimidación en el trabajo. Esto se condice con lo hipotetizado en este estudio respecto al efecto indirecto del liderazgo en la salud mental, mediado por la VL.

Finalmente, cabe señalar que se consideró el distrés como proxy de problemas de salud mental, dado que es ampliamente utilizado en la literatura sobre VL (Attell et al., 2017; Demir \& Rodwell, 2012; Malik et al., 2018; Nielsen et al., 2013; Reknes et al., 2017; Sperduti et al., 2018; Tonso et al., 2016; van der Velden et al., 2016; Zafar et al., 2016). En este estudio el distrés es considerado desde el concepto de Kessler et al. (2002), esto es, como el malestar psicológico compuesto por síntomas de depresión, inquietud, fatiga, sentimiento de culpa y ansiedad.

\section{Esta Investigación}

El modelo propuesto — del cual se derivan las hipótesis que se plantearán a continuación- puede observarse en la Figura 1. Este modelo propone que los riesgos psicosociales (ISOSTRAIN y desbalance esfuerzo-recompensa) y la exposición a liderazgo tiránico son antecedentes de la VL y problemas de salud mental (distrés). A su vez, el modelo propone efectos indirectos de los riesgos psicosociales en el distrés, mediados por la VL. Es decir, parte de la razón por la que los riesgos psicosociales y el liderazgo llevarían a peores niveles de distrés se debería a que influyen en generar mayores probabilidades de ser víctimas de VL por parte de los/as trabajadores/as. Estudios previos proveen evidencia de un efecto indirecto, mediado por VL, del liderazgo en la salud mental (Spence Laschinger et al., 2012). A su vez, Balducci et al. (2011) encontraron en una muestra de trabajadores italianos que la intimidación mediaba la relación entre las demandas de trabajo y los síntomas de trastorno por estrés postraumático.

Figura 1

Modelo Propuesto de Relaciones entre Riesgos Psicosociales, Liderazgo, Violencia Laboral y Distrés

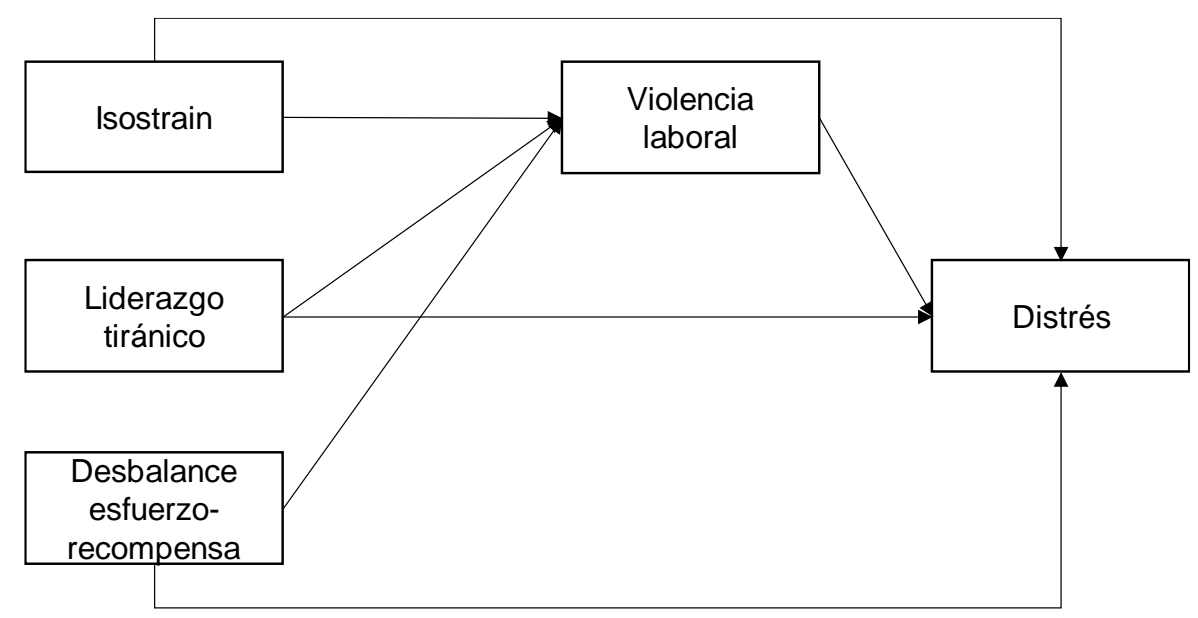


Del modelo propuesto se desprenden las siguientes hipótesis:

Hipótesis 1: El nivel de exposición a VL se relaciona directamente con el nivel de distrés.

Hipótesis 2: La exposición a ISOSTRAIN se relaciona directamente con la exposición a VL (H2a) y con el nivel de distrés (H2b). El efecto del ISOSTRAIN en el distrés está en parte mediado por la VL (H2c).

Hipótesis 3: La exposición a desbalance esfuerzo-recompensa se relaciona directamente con la VL (H3a) y con el nivel de distrés (H3b). El efecto del desbalance esfuerzo-recompensa en el distrés está en parte mediado por la VL (H3c).

Hipótesis 4: La exposición a liderazgo tiránico se relaciona directamente con el nivel de exposición a VL (H4a) y con el nivel de distrés (H4b). El efecto de la exposición a liderazgo tiránico en el distrés se encuentra en parte mediado por la exposición a VL (H4c).

\section{Método}

\section{Participantes}

Los participantes fueron 1023 funcionarios (302 hombres, 721 mujeres) de tres hospitales públicos chilenos de alta complejidad, correspondiente al 10,5\% de la dotación total de dichos hospitales $(N=9.736$; Palma-Contreras \& Ansoleaga, 2020). Los hospitales fueron elegidos de manera intencionada por tratarse de aquellos con la mayor dotación de personal de tres importantes regiones de Chile. Las regiones no son identificadas para proteger el anonimato de los hospitales, como fue solicitado por los comités de ética de estos. Los funcionarios fueron seleccionados mediante un muestreo segmentado por sexo y estamento (administrativo, auxiliar, técnico, personal médico, otro profesional no médico y directivo). En uno de los hospitales se realizó un muestreo estratificado, es decir, para cada segmento se seleccionó al azar a los participantes desde un listado. En los otros dos hospitales se efectuó un muestreo por cuotas por sexo y estamento, dado que se contaba con información sobre la composición de los estamentos, pero no se logró acceso al marco muestral. Cabe señalar que esta muestra ha sido utilizada para otros análisis publicados en un artículo de Palma-Contreras y Ansoleaga (2020). La descripción de la muestra puede verse en la Tabla 1. La muestra se encuentra compuesta en mayor medida por mujeres $(70,5 \%)$, personas de 18 a 30 años $(27,8 \%)$ y de 31 a 40 años $(28,1 \%)$, de estamentos técnicos $(33,6 \%)$ y a contrata $(47,9 \%)$.

Tabla 1

Descripción de la Muestra

\begin{tabular}{lccc}
\hline \multicolumn{1}{c}{ Variable } & Mujeres $n(\%)$ & Hombres $n(\%)$ & Total $n(\%)$ \\
\hline Total & $721(70,5)$ & $302(29,5)$ & $1023(100,0)$ \\
Rangos de edad & & & \\
$\quad$ 18-30 & $206(29,1)$ & $74(24,7)$ & $280(27,8)$ \\
$31-40$ & $193(27,3)$ & $90(30,0)$ & $283(28,1)$ \\
$41-50$ & $152(21,5)$ & $62(20,7)$ & $214(21,2)$ \\
$51-60$ & $126(17,8)$ & $50(16,7)$ & $176(17,5)$ \\
$\quad 61$ y más & $31(4,4)$ & $24(8,0)$ & $55(5,5)$ \\
Estamentos & & & \\
$\quad$ Administrativos & $81(11,3)$ & $20(6,6)$ & $101(9,9)$ \\
Auxiliares & $59(8,2)$ & $68(22,6)$ & $127(12,4)$ \\
Técnicos & $292(40,6)$ & $51(16,9)$ & $343(33,6)$ \\
Personal médico & $93(12,9)$ & $111(36,9)$ & $204(20,0)$ \\
Otros profesionales no médicos & $195(27,1)$ & $51(16,9)$ & $246(24,1)$ \\
Tipo de contrato & & & \\
Titular (indefinido) & $211(29,4)$ & $83(27,7)$ & $294(28,9)$ \\
Contrata (plazo fijo anual) & $336(46,8)$ & $152(50,7)$ & $488(47,9)$ \\
Honorarios & $64(8,9)$ & $17(5,7)$ & $81(8,0)$ \\
Otro & $107(14,9)$ & $48(16,0)$ & $155(15,2)$ \\
\hline
\end{tabular}

Fuente. Palma-Contreras \& Ansoleaga, 2020, p. 3. Los casos faltantes son por no respuesta. 


\section{Instrumentos}

\section{Variables Dependientes}

Violencia Laboral. Se midió con el Negative Act Questionnaire-Revised (NAQ-R), adaptado y validado en Chile por González (2019). Este instrumento evalúa la presencia de conductas negativas en el trabajo mediante una escala Likert de 22 ítems con cinco alternativas de respuesta: Nunca (0), Alguna vez (1), Al menos una vez al mes (2), Al menos una vez a la semana (3), A diario (4). Un ejemplo de ítem es: "Le han gritado o ha sido objeto de enfados espontáneos". El puntaje bruto se obtiene de la suma simple de los ítems. Un mayor puntaje indica más exposición a violencia laboral. La consistencia interna, evaluada con alfa de Cronbach, es de 0,93 (González, 2019).

Distrés. Se midió con la Escala de Kessler (K6), screening de distrés psicológico (Kessler et. al., 2002), validada en Chile por Ansoleaga et al. (2013). Corresponde a una escala Likert de 6 ítems con cinco alternativas de respuesta, desde Nunca (0) hasta Siempre (4). Los ítems consultan la frecuencia con que durante los últimos 30 días se ha sentido "Nervioso/a"; "Desesperado/a"; "Descontrolado/a o fuera de sí"; "Tan deprimido/a que nada le puede hacer sonreír"; "Que todo es un esfuerzo" y "Bueno para nada". El puntaje se obtiene de la suma simple de las respuestas a los ítems. A mayor puntaje mayor presencia del atributo, en este caso, mayor distrés. La consistencia interna, evaluada con alfa de Cronbach, es de 0,88 (Ansoleaga et al., 2013).

\section{Variables Independientes}

Exposición a Liderazgo Tiránico. Se midió con cuatro ítems de la Escala de Liderazgo Destructivo, adaptada y validada en Chile por González (2019). Posee cuatro categorías de respuesta desde Nunca (0) hasta Casi siempre (3). Por ejemplo: "Ha sido humillado o ridiculizado en relación a su trabajo". Las respuestas se dicotomizaron, considerando como expuestos a liderazgo tiránico a quienes contestaron Bastante a menudo o Casi siempre a alguna de las preguntas de la escala y como no expuestos a liderazgo tiránico a los demás (Aasland et al., 2010; Palma-Contreras \& Ansoleaga, 2020).

Desbalance Esfuerzo-Recompensa. Se midió con el Effort-Reward Imbalance Questionnaire (ERI; Siegrist et. al., 2009), que fue validado en Chile por Ansoleaga et al. (2013), obteniendo un $a=0,72$. Esta escala está compuesta por tres ítems de esfuerzo y siete de recompensa, con cuatro categorías de respuesta, desde Muy en desacuerdo (1) hasta Muy de acuerdo (4). Un ejemplo de ítem esfuerzo es: "En el último tiempo tengo cada vez más trabajo" y uno de ítem recompensa es: "Si pienso en todo el trabajo y esfuerzo que he realizado, mi sueldo me parece adecuado". Se suman por separado los puntajes de esfuerzos y recompensas. Se consideró como desbalance los puntajes superiores a 1 en la siguiente fórmula: $\Sigma$ puntaje esfuerzo / $\Sigma$ puntaje recompensas x 0,4285. El valor 0,4285 en la fórmula corresponde a la división de 3/7 (correspondiente a los 3 ítems de esfuerzo y los 7 de recompensa), permitiendo ponderar la diferencia respecto de la cantidad de ítems (Ansoleaga, 2015; Palma-Contreras \& Ansoleaga, 2020).

ISOSTRAIN. Se refiere a la presencia simultánea de altas demandas psicológicas, baja latitud decisional (tensión psíquica) y bajo soporte social (Karasek \& Theorell, 1990). Se midió con el Job Questionnaire de Karasek et al. (1998), utilizando la versión validada en Chile por Ansoleaga et al. (2013), en la que obtuvo un $a=0,74$. El instrumento corresponde a una escala Likert de 10 ítems con cuatro alternativas de respuesta, desde Muy en desacuerdo (1) hasta Muy de acuerdo (4). Ejemplos de ítems de demanda psicológica: "Mi trabajo exige trabajar muy rápido" o "Me piden hacer una cantidad excesiva de trabajo". En total son 9 ítems, con un puntaje teórico de 36. Se consideró con altas demandas psicológicas los puntajes mayores a 21. Un ejemplo de latitud decisional es: "Tengo libertad de decidir cómo hacer mi trabajo". En total son 5 ítems, con un puntaje teórico de 20. Se consideró con baja latitud decisional los puntajes iguales o menores a 13. Un ejemplo de apoyo de superiores es: "Mi jefe inmediato presta atención a lo que yo digo (considera mi punto de vista) ", y de apoyo de pares es: "Mis colegas facilitan la realización de mi trabajo". En total son 6 ítems de apoyo social, con un puntaje teórico de 30. Se consideró con bajo apoyo social los puntajes iguales o menores a 17 . Se trabajó con la variable dicotómica ISOSTRAIN, construida considerando como presencia de ISOSTRAIN a los sujetos que presentaban simultáneamente altas demandas psicológicas, baja latitud decisional y bajo soporte social (Karasek \& Theorell, 1990; Palma-Contreras \& Ansoleaga, 2020). 


\section{Variables de Control}

Se usaron como variables de control varias mencionadas en la literatura como relevantes para predecir la VL y el distrés. Se incorporaron las siguientes: sexo (hombre/mujer), edad, estamento profesional/no profesional, tipo de horario (diurno/no diurno), tipo de contrato (no precario/precario), estrechez económica (con o sin estrechez) y vulnerabilidad laboral (con o sin vulnerabilidad). Esta elección tuvo dos motivos: primero, porque "los patrones poblacionales de buena y mala salud [mental] reflejan las distribuciones poblacionales de deprivación y privilegio" (Krieger, 2001, p. 1); segundo, porque el desequilibrio de poder entre el perpetrador y la víctima es una característica imprescindible en la VL (Beal, 2011, citado en Díaz Berr et al., 2017; Escartín et al., 2012). Las variables elegidas dan cuenta de la posición que ocupan los sujetos en la sociedad general y ello influye en la desigualdad de poder entre el perpetrador y la víctima, puesto que dicha desigualdad deriva no solo de la jerarquía ocupacional, sino también de las desigualdades y discriminaciones existentes en la sociedad (Beal, 2011, citado en Díaz Berr et al., 2017; Salin, 2003). A continuación, se describen en detalle estas variables de control.

Vulnerabilidad Laboral. La vulnerabilidad laboral se consideró como un indicador de precariedad laboral. Puede definirse como los "temores que influyen en cómo los trabajadores perciben su propia capacidad para interactuar con empleadores y supervisores" (Casas Becerra, 2016, p. 181) que generan la percepción de indefensión e incapacidad para ejercer los derechos laborales (Amable, 2006). La vulnerabilidad laboral se encuentra asociada al concepto de inseguridad laboral — combinación de percepción de amenaza, impotencia e incertidumbre sobre el futuro (Greenhalgh \& Rosenblatt, 1984; De Witte, 1999)—, concepto ampliamente vinculado con resultados de salud mental adversos (Llosa et al., 2018; Park \& Ono, 2017) y presencia de VL (Glambek et al., 2014; Park \& Ono, 2017; Spagnoli \& Balducci, 2017). Se midió con una escala de 7 ítems. Un ejemplo de ítem es: "Se siente preocupado/a de que le cambien las condiciones de su salario", con cuatro alternativas de respuesta tipo Likert, desde Muy en desacuerdo (1) hasta Muy de acuerdo (4) (Amable, 2006). Siguiendo el criterio de expertos, se consideró con vulnerabilidad laboral a quiénes contestaron positivamente a tres o más situaciones de vulnerabilidad laboral, con la finalidad de contar con un grupo de expuestos y otro de no expuestos.

Estrechez Económica. La estrechez económica se midió desde una percepción subjetiva del trabajador/a acerca de tener dificultades económicas a fin de mes (Ansoleaga Moreno, 2013). La estrechez económica ha sido asociada a peores resultados de salud mental (Han et al., 2017), poniendo al trabajador/a en una posición de menor poder subjetivo, lo que influye en el riesgo de ser víctima de VL (Tsuno et al., 2015). Se consultó con la pregunta: "...pensando en el total de ingresos mensuales de su hogar, este:" Les alcanza bien, pudiendo ahorrar (1), Les alcanza justo, sin grandes dificultades (2), No les alcanza, tienen algunas dificultades (3), No les alcanza, tienen grandes dificultades (4). La variable se dicotomizó respecto de quienes presentan dificultades económicas a fin de mes (alternativas 3 y 4) y quienes no las presentan (alternativas 1 y 2) (Ansoleaga Moreno, 2013; Palma-Contreras \& Ansoleaga, 2020).

Tipo de Contrato. Se consideró el tipo de contrato de los trabajadores/as, dado que los empleados con contrato temporal o informal han mostrado estar en mayor riesgo de sufrir VL (Keuskamp et al., 2012; Notelaers et al., 2011), mayor riesgo de requerir tratamiento médico por enfermedad mental (Moscone et al., 2016) y efectos nocivos en su salud y bienestar (Julià et al., 2017). Se midió con la pregunta "¿Qué tipo de contrato tiene en este hospital?". Las alternativas de respuesta fueron: Planta (1), Contrata (2), Honorarios (3) Otro (4). Se consideró a aquellos/as trabajadores/as con contratos a honorarios u otro como teniendo un contrato precario y a los/as trabajadores/as de planta o a contrata como no teniendo contratos precarios (Palma-Contreras \& Ansoleaga, 2020).

Horario. La literatura muestra que los turnos de noche y los horarios irregulares aumentan el riesgo de lesiones y el autorreporte como víctima de VL (Al-Azzam et al., 2017; Ariza-Montes et al., 2013; Fischer et al., 2019), mientras que los trabajadores/as por turno tienen mayor riesgo de tener bajo control sobre el trabajo, altas demandas emocionales y bajo apoyo social (Fischer et al., 2019). Se midió con la pregunta "Respecto a su horario de trabajo, ¿este es?", cuyas alternativas de respuesta fueron: Solo diurno (de día) (1), Solo nocturno (de noche) (2), En turnos rotativos día-noche (3), En turnos por ciclos (dias de trabajo y de descanso, por ej.: 10x5; $4 \times 3$ (4), Otro (5). Se trabajó con una variable dicotómica, incluyendo como horario no diurno las alternativas 2,3 , 4 y 5 y como diurno, la 1. 
Profesional/No Profesional. Se preguntó por el estamento al que pertenece el participante. Las alternativas de respuesta fueron: Médico (1), Odontólogo (2), Químico o bioquímico (3), Enfermera/o universitaria /o (4), Otro profesional no médico (5), Técnico paramédico o de enfermería (6), Otros técnicos (7), Administrativo (8), Auxiliar (9). Se clasificó dentro del estamento profesional las alternativas 1 a 5 y en el estamento no profesional las alternativas restantes (6 a 9).

Sexo. Se consideró el sexo, dado que las mujeres están más expuestas a VL (Centro de Estudios de la Mujer \& Universidad de Ottawa, 2011; Seun-Fadipe et al., 2019; Yada et al., 2014) y ser víctima de esta se asocia a más problemas de distrés en mujeres (Attell et al., 2017; Zhao et al., 2018). Se midió con una pregunta de autorreporte categórica: Hombre (0) y Mujer (1).

Edad. Se consideró la edad dado que, según la literatura, las personas de menor edad están expuestas a mayor VL (Obeidat et al., 2018; Radey \& Wilke, 2018; Seun-Fadipe et al., 2019; Zhao et al., 2018). En la medida en que los trabajadores/as envejecen la carga de trabajo, el conflicto y la ambigüedad de rol pierden importancia, mientras que el reconocimiento resulta relativamente más importante (López-Cabarcos et al., 2017). Se midió por medio de un autorreporte de los años de vida.

\section{Procedimiento}

Los hospitales fueron invitados a participar mediante contacto por correo electrónico con la dirección de los establecimientos, los departamentos de personas y las unidades docente-asistenciales.

El estudio recibió la aprobación de los comités de ética de la Facultad de Psicología de la Universidad Diego Portales y de los tres hospitales de la muestra ( $\mathrm{N}^{\circ} 028-2017$ y NO2886). Los participantes leyeron y firmaron un consentimiento informado resguardando confidencialidad y voluntariedad.

La recolección de información se llevó a cabo entre mayo y noviembre de 2018, mediante un cuestionario autoadministrado en papel. El orden de las variables fue el siguiente: sexo, estamento, tipo de contrato, demandas psicológicas, latitud decisional, violencia laboral, esfuerzos, recompensas, apoyo social, liderazgo, vulnerabilidad laboral, distrés y estrechez económica. En dos hospitales los funcionarios contestaron el cuestionario en su puesto de trabajo y en el tercer hospital fueron convocados en un espacio común (auditorio) para hacerlo, todo ello dentro de su horario laboral. Se aseguró la confidencialidad, ya que los encuestados contestaron en privado y el cuestionario era entregado y retirado por el encuestador. La aplicación del cuestionario tuvo una duración promedio de 20 minutos.

\section{Análisis de Datos}

Primero se realizaron análisis descriptivos para dar cuenta de la distribución de los riesgos psicosociales, la VL y el distrés entre los trabajadores/as encuestados/as Luego se realizaron análisis bivariados a través del análisis de correlaciones $r$ de Pearson entre VL, distrés e ISOSTRAIN y, mediante la prueba $t$ de Student para muestras independientes y asumiendo varianzas desiguales, las diferencias en la VL y el distrés, según desbalance esfuerzo-recompensa y liderazgo tiránico. Se utilizaron pruebas $t$ de Student que asumen varianzas desiguales, dado que en todos los casos la prueba de igualdad de varianzas de Levene mostró varianzas significativamente distintas entre los grupos al $95 \%$ de confianza $(p<0,001)$. El tamaño del efecto fue calculado a través de una $d$ de Cohen de Welch, la cual no asume varianzas iguales. Por último, se realizó un análisis de senderos por medio del paquete lavaan en RStudio 1.2.1335 (Rosseel, 2012) para evaluar el modelo presentado en la Figura 1. Se trabajó con variables observadas, dado que la mayoría de los constructos se miden con escalas sumatorias. Se consideraron como variables dependientes el distrés y la VL, mientras que como variables independientes se consideraron el ISOSTRAIN, desbalance esfuerzo-recompensa y liderazgo tiránico. Las variables de control mencionadas arriba se agregaron como variables control en los modelos que predicen el distrés y la VL. Se utilizó un método de estimación de máxima verosimilitud, utilizando errores estándar robustos, dado que los puntajes de las variables dependientes no se distribuyen de manera normal. El uso de errores robustos permite generar estimadores no sesgados de los coeficientes sin asumir homocedasticidad de los residuos. Se presentan coeficientes estandarizados para las variables medidas a nivel de escala. El ajuste del modelo fue evaluado según criterios convencionales (Prueba de Chi Cuadrado no significativa, CFI > 0,95 y RMSEA < 0,05). 


\section{Resultados}

La prevalencia en las variables consideradas aparece en la Tabla 2, extraída del artículo anterior ya publicado (Palma-Contreras \& Ansoleaga, 2020). Más allá de lo expuesto en la Tabla 2, respecto a la VL, el puntaje máximo de la prueba fue 79 y el mínimo fue 0 , la media fue de 11,57 puntos y la mediana, 8. Existen distintos criterios para definir sobre qué puntaje un/a trabajador/a es catalogado/a como expuesto/a a VL. De acuerdo al criterio de Notelaers y Einarsen (2013), que establece como puntaje de corte 32 puntos en el NAQ-R, $54(5,3 \%)$ encuestados serían considerados acosados de vez en cuando o víctimas de acoso. Sin embargo, siguiendo el criterio de González (2019), que utilizó el mismo método que Notelaers y Einarsen (2013), quien sugiere establecer puntos de corte empíricos definido en 9 puntos en una muestra de trabajadores/as chilenos/as, $488(47,7 \%)$ de los encuestados reportarían haber sido acosados de vez en cuando o haber sido víctimas de acoso. Con respecto al distrés, el puntaje promedio de la Escala K6 fue 3,62, con un mínimo de 0 y un máximo de 24 puntos, el puntaje teórico máximo.

Tabla 2

Prevalencia de Categorías de Variables Principales

\begin{tabular}{lcccc}
\hline \multicolumn{1}{c}{ Categoría de variable } & Hombres & Mujeres & $p$ & Total $n(\%)$ \\
\hline Violencia laboral & $43(15)$ & $101(14,6)$ & 0,863 & $144(14,7)$ \\
Riesgos psicosociales & & & & \\
$\quad$ Altas demandas psicológicas & $134(45,3)$ & $360(51,6)$ & 0,066 & $494(49,7)$ \\
$\quad$ Baja latitud decisional & $90(30,2)$ & $268(37,9)$ & 0,021 & $358(35,6)$ \\
JOBSTRAIN & $44(15,0)$ & $136(19,7)$ & 0,076 & $180(18,3)$ \\
Bajo soporte social & $186(61,6)$ & $436(61,0)$ & 0,855 & $622(61,2)$ \\
ISOSTRAIN & $38(12,9)$ & $114(16,6)$ & 0,146 & $152(18,6)$ \\
$\quad$ Altos esfuerzos & $113(37,7)$ & $320(44,8)$ & 0,037 & $433(42,7)$ \\
$\quad$ Bajas recompensas & $137(46,4)$ & $378(54,2)$ & 0,025 & $515(51,9)$ \\
$\quad$ Desbalance esfuerzo recompensa & $150(51,5)$ & $398(58,1)$ & 0,059 & $548(56,1)$ \\
Dimensiones organizacionales & & & & \\
$\quad$ Exposición a liderazgo tiránico & $43(14,4)$ & $107(15)$ & 0,06 & $150(14,9)$ \\
Variables de control & & & & \\
$\quad$ Estrechez económica & $59(19,6)$ & $176(24,6)$ & 0,085 & $235(23,1)$ \\
$\quad$ Vulnerabilidad laboral & $111(37,0)$ & $304(42,6)$ & 0,099 & $415(40,9)$ \\
$\quad$ Contrato precario & $65(21,7)$ & $171(23,2)$ & 0,459 & $236(23,2)$ \\
Problemas de salud mental & & & & \\
$\quad$ Distrés elevado & $45(15,2)$ & $127(18,1)$ & 0,265 & $172(17,2)$ \\
\hline
\end{tabular}

Fuente. Palma-Contreras \& Ansoleaga, 2020, p. 7.

La Tabla 3 muestra las correlaciones bivariadas entre VL, distrés e ISOSTRAIN. Aquí es posible observar que mayores niveles de VL se asocian con mayores niveles de distrés y mayores niveles de ISOSTRAIN. A su vez, se observa una relación directa, aunque menor, entre distrés e ISOSTRAIN.

Tabla 3

Correlaciones entre Violencia Laboral, Distrés e ISOSTRAIN

\begin{tabular}{lrrccc}
\hline & \multicolumn{1}{c}{$M$} & \multicolumn{1}{c}{$D E$} & Violencia laboral & Distrés & ISOSTRAIN \\
\hline Violencia laboral & 11,57 & 11,77 & 1,00 & & \\
Distrés & 3,62 & 3,80 & $0,47^{*}$ & 1,00 & \\
ISOSTRAIN & 0,15 & 0,36 & $0,37^{*}$ & $0,20^{*}$ & 1,00 \\
\hline
\end{tabular}

${ }^{*} p<0,01$ 
La Tabla 4 resume los promedios (M) y desviaciones estándar (DE) de VL y distrés para cada grupo de las variables desbalance esfuerzo-recompensa y liderazgo tiránico. Así, se observan mayores promedios de VL y distrés en personas con alto desbalance esfuerzo-recompensa, comparado con personas con niveles bajos, y en personas que dicen sufrir un liderazgo tiránico, comparado con personas con ausencia de esta forma de liderazgo. Estas diferencias son significativas al 95\% de confianza. A su vez, el tamaño del efecto, estimado a través de una $d$ de Cohen para varianzas desiguales, muestra efectos grandes (sobre 0,8) para los niveles de violencia laboral según desbalance esfuerzo-recompensa y la exposición a liderazgos tiránicos y efectos moderados para el caso del distrés (valores en torno a 0,5).

\section{Tabla 4}

Media de Violencia Laboral y Distrés según Desbalance Esfuerzo-Recompensa y Liderazgo Tiránico

\begin{tabular}{|c|c|c|c|c|c|}
\hline & & \multicolumn{2}{|c|}{ Violencia laboral } & \multicolumn{2}{|c|}{ Distrés } \\
\hline & & $M$ & $D E$ & $M$ & $D E$ \\
\hline \multirow{3}{*}{$\begin{array}{l}\text { Desbalance } \\
\text { esfuerzo- } \\
\text { recompensa }\end{array}$} & Bajo & 6,40 & 7,42 & 2,35 & 2,68 \\
\hline & Alto & 15,60 & 12,89 & 4,62 & 4,23 \\
\hline & & \multicolumn{2}{|c|}{$\begin{array}{c}t(935)=-12,94, p<0,001 \\
d=-0,875\end{array}$} & \multicolumn{2}{|c|}{$\begin{array}{c}t(958)=-9,62, p<0,001 \\
d=-0,641\end{array}$} \\
\hline \multirow{2}{*}{$\begin{array}{l}\text { Liderazgo } \\
\text { tiránico }\end{array}$} & Ausente & 9,89 & 9,83 & 3,32 & 3,44 \\
\hline & Presente & \multicolumn{2}{|c|}{$\begin{array}{c}t(968)=-11,38, p<0,001 \\
d=-0,838\end{array}$} & \multicolumn{2}{|c|}{$\begin{array}{c}t(988)=-6,19, p<0,001 \\
d=-0,476\end{array}$} \\
\hline Total & & 11,57 & 11,77 & 3,62 & 3,80 \\
\hline
\end{tabular}

Con respecto al análisis de senderos, en una primera instancia se incluyeron todos los efectos directos del ISOSTRAIN, desbalance esfuerzo-recompensa y liderazgo tiránico en la VL y el distrés, así como el efecto directo de la VL en el distrés (ver Figura 1). Este modelo asumía que el ISOSTRAIN, el desbalance esfuerzorecompensa y el liderazgo tiránico tendrían efectos tanto directos como indirectos en el distrés. Sin embargo, el análisis mostró que ni el ISOSTRAIN ni el liderazgo tiránico tenían efectos directos en el distrés al 95\% de confianza. Por lo tanto, se estimó un nuevo modelo que eliminaba estos efectos directos y solamente dejaba los efectos indirectos mediados por la VL (ver Figura 2). Una vez obtenido el modelo, se procedió a estimar los efectos indirectos de ISOSTRAIN, liderazgo tiránico y desbalance esfuerzo-recompensa en el distrés.

El análisis de senderos mostró un buen ajuste: $\chi^{2}(2, n=902)=0,094, p=0,954$; CFI $=1,000$, RMSEA $=0,000$. Sin embargo, es necesario considerar que el modelo se encuentra casi saturado, por lo que valores muy altos de CFI y muy bajos de RMSEA son esperables.

Los resultados proveen evidencia a favor de la Hipótesis 1, con un efecto positivo y significativo de la VL en el distrés. Con respecto a la Hipótesis 2, se observó que a mayor ISOSTRAIN, mayores niveles de VL (H2a) $\mathrm{y}$, por medio de esta, un mayor distrés (H2c; $B=0,08, p<0,01)$. Sin embargo, a diferencia de lo hipotetizado (H2b), el efecto de ISOSTRAIN en distrés fue capturado completamente por su efecto en la VL, sin observarse un efecto directo. Los resultados también son consistentes con la Hipótesis 3, dado que el desbalance esfuerzorecompensa se relaciona positiva y significativamente con la VL (H3a) y con el nivel de distrés (H3b), presentando también un efecto indirecto en el distrés, mediado por la VL (H3c; $B=0,17, p<0,01$ ). Entonces, a medida que aumenta el desbalance esfuerzo-recompensa el distrés aumenta en 0,36 desviaciones estándar $(p<0,01)$. Por último, con respecto a la Hipótesis 4, se evidenció que las personas expuestas a liderazgo tiránico presentan mayores niveles de VL que las no expuestas (H4a) y, por medio de esto, mayores niveles de distrés (H4c; $B=0,22, p<0,01)$. Sin embargo, no se encontró un efecto directo del liderazgo en el distrés (H4b). El modelo completo predice el 34,9\% de la varianza en VL y el 28,5\% de la varianza en distrés. 
Figura 2

Resultados del Modelo de Senderos que Predice Violencia Laboral y Distrés

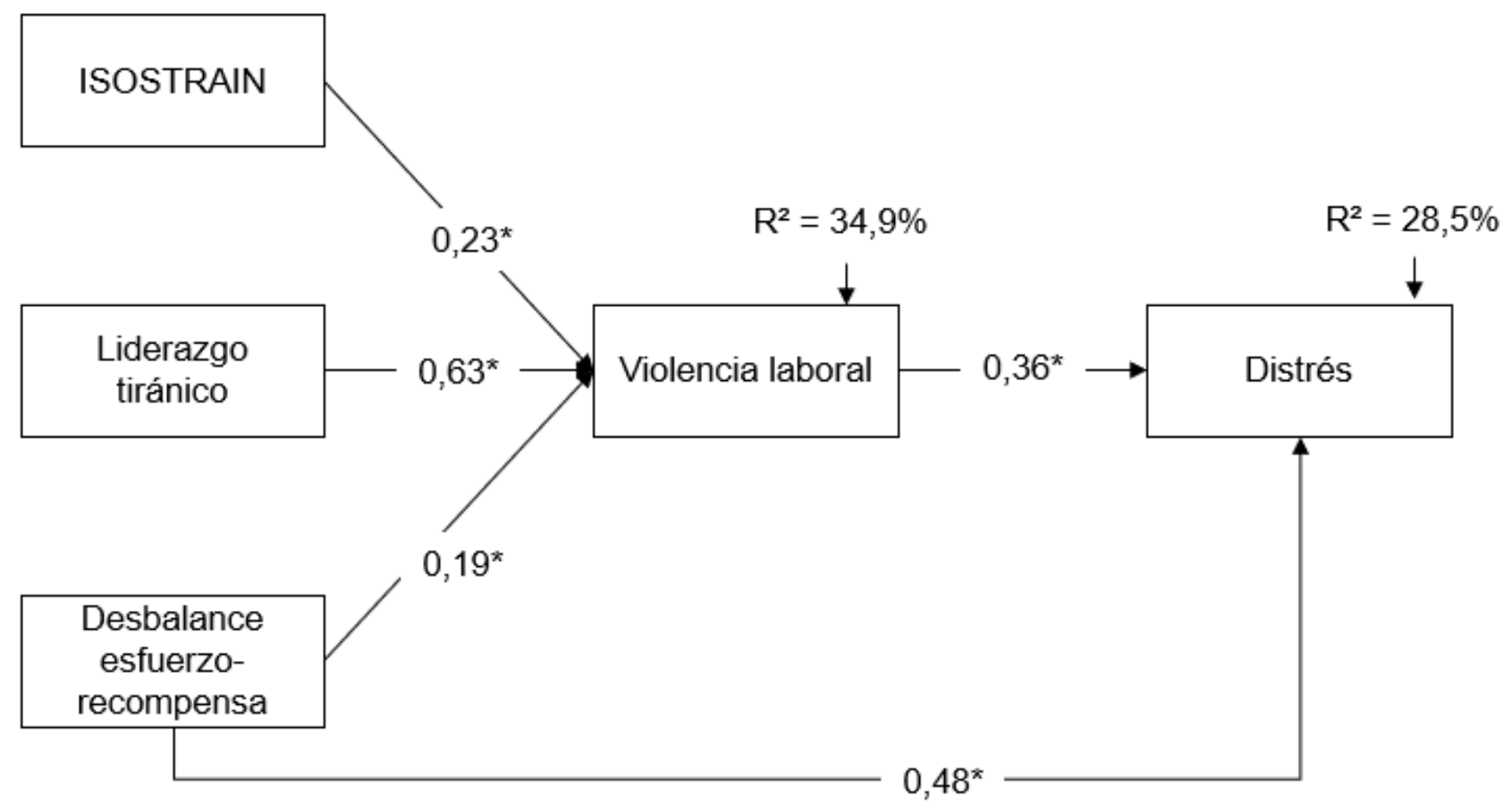

Nota. El modelo está controlado por sexo, edad, estrechez económica, vulnerabilidad laboral, precariedad del contrato, horario no diurno y estamento no profesional en cada uno de los niveles. Se presentan efectos estandarizados. Los efectos indirectos del desbalance esfuerzo-recompensa $(B=0,17, p<0,01)$, de ISOSTRAIN $(B=0,08, p<0,01)$ y de liderazgo tiránico $(B=0,22, p<0,01)$ en distrés son todos significativos y positivos. $\mathrm{R}^{2}$ corresponde al porcentaje de varianza de la variable dependiente que es explicada por el modelo. * $p<0,01$

\section{Discusión}

En el presente artículo se hipotetizó que la VL cumpliría un rol mediador entre, por una parte, los riesgos psicosociales y el liderazgo, y, por otra parte, el distrés. La principal contribución de este trabajo consiste en proveer evidencia a favor del rol mediador que tiene la VL en la relación del liderazgo tiránico y los riesgos psicosociales con los resultados en distrés.

$\mathrm{Al}$ igual que en estudios internacionales previos con trabajadores hospitalarios (Baby et al., 2014; Dement et al., 2014; Einarsen \& Nielsen, 2015; Itzhaki et al., 2015; Magnavita, 2014; Pien et al., 2015; Rodwell \& Demir, 2012; Zhao et al., 2018), este estudio corroboró la existencia de una relación positiva entre exposición a VL y distrés psicológico. Particularmente, dicho hallazgo coincide con estudios internacionales, tales como el de Demir y Rodwell (2012), quienes encontraron que la intimidación se relaciona con un aumento del nivel de distrés, el estudio de Nielsen et al. (2013), quienes concluyeron que la exposición a acoso laboral es predictora de distrés posterior, el de Reknes et al. (2017) quienes reportan que la VL externa y la exposición a conductas de acoso laboral se perciben como factores de estrés, y los de van der Velden et al. (2016) y Sperduti et al. (2018), que evidenciaron que la VL se relaciona con estrés. A nivel nacional, dicho hallazgo coincide con lo reportado por Ansoleaga et al., (2020), quienes en una muestra de 1995 trabajadores/as chilenos/as de diversos sectores laborales encontraron asociación entre exposición a VL y distrés elevado en hombres.

Por otra parte, en este estudio se encontró que los riesgos psicosociales se relacionan positivamente con la exposición a VL. Este resultado coincide con la evidencia previa disponible a nivel internacional (Arnetz et al., 2018). Adicionalmente, a nivel nacional Ansoleaga (2015) encontró que trabajadores sanitarios poseen mayor probabilidad de reportar distrés elevado cuando indican exposición a riesgos psicosociales. Con respecto al efecto de los riesgos psicosociales en el distrés, el efecto del ISOSTRAIN (esto es, presencia simultánea de altas demandas psicológicas, baja latitud decisional y bajo soporte social) fue completamente 
mediado por la VL, mientras que el efecto del desbalance esfuerzo-recompensa mantuvo un efecto directo, además del efecto mediado por VL. Estos resultados extienden la evidencia de estudios internacionales previos (Cheng \& Cheng, 2017; Russo et al., 2019; Salas et al., 2015) que establecen una relación entre riesgos psicosociales, VL y distrés, esta vez encontrando evidencia acerca del rol mediador de la VL en vez de simplemente tratarla como variable de control.

El hecho de que el efecto del ISOSTRAIN en el distrés se encuentre totalmente mediado por la VL (al controlar por el desbalance esfuerzo-recompensa y el liderazgo) sugiere que, en esta muestra, no se observa un efecto del ISOSTRAIN en el distrés que no resulte del aumento en los niveles de VL observados. Es decir, no habría aquí evidencia a favor de otros mecanismos por medio de los cuales el ISOSTRAIN pudiera aumentar el distrés, tales como la alta tensión psíquica y el bajo apoyo social. Esto puede deberse a que el ISOSTRAIN se refiere a modos de organizar el trabajo a los que se encuentran expuestos de manera generalizada los trabajadores de los hospitales, entre otras razones, por la implementación de estilos de gestión característicos de la nueva gestión pública que entran en conflicto con el modelo de vocación de servicio público, y, también, debido a que en el sector salud se observó estilos de relación altamente jerárquicos que son asumidos como modos naturales de relación en el trabajo y, en consecuencia, muchas veces no son percibidos teniendo la intención de dañar a los trabajadores y por ello, se ha hipotetizado que es posible que la exposición a ISOSTRAIN no afecte en el distrés de los trabajadores.

En este estudio se observa una relación dosis-respuesta entre la intensidad de la exposición a VL y el aumento de los niveles de distrés de los funcionarios, hallazgo que coincide con lo reportado en estudios previos que incluyen la VL como variable independiente, unida a otros riesgos psicosociales, y la relacionan con indicadores de problemas de salud mental (Aronsson et al., 2019; Carretero Domínguez et al., 2011; Cheng \& Cheng, 2017; Niedhammer et al., 2016; Russo et al., 2019; Salas et al., 2015). Como señaló Franco (2015), la existencia de relación entre VL y distrés se puede deber a que las víctimas de VL tienden a percibir que se trata de conductas intencionales y, en sentido contrario, la inexistencia de relación directa entre ISOSTRAIN y distrés puede vincularse a que este riesgo psicosocial es parte de las condiciones generales del trabajo en salud y que se encuentran presentes de manera generalizada - y en ocasiones naturalizada-, afectando a la mayoría de los/as trabajadores/as de la salud. Así, es posible hipotetizar que la atribución de intencionalidad juega un rol importante en el aumento del distrés de las/os trabajadores (Franco, 2015). Estudios futuros podrían incluir mediciones de percepciones de intencionalidad para evaluar esta hipótesis.

El hallazgo de este estudio referente a la mediación de la VL en la relación entre el ISOSTRAIN y el nivel de distrés tiene ciertas similitudes con lo reportado por Balducci et al. (2011), quienes encontraron que la intimidación media la relación entre las demandas de trabajo y los síntomas de trastorno por estrés postraumático. Asimismo, la asociación directa entre desbalance esfuerzo-recompensa y VL se relaciona con la evidencia previa de estudios como el de Feijó et al. (2019), que indican que el desequilibrio esfuerzorecompensa aumenta la prevalencia de VL interna. En este sentido, cabe señalar que la falta de reciprocidad entre los esfuerzos invertidos y las recompensas recibidas - perspectivas de promoción, seguridad en el empleo, estima relacionada con el desempeño - provoca fuertes emociones negativas (Siegrist et al., 2009) y ello puede conducir a conflictos interpersonales que, si no se resuelven adecuadamente, resulten en violencia tanto entre trabajadores como de parte de los usuarios, esto último por el deterioro de la calidad de la atención que puede producirse.

La asociación entre desbalance esfuerzo-recompensa y distrés confirma la relación previamente reportada por la evidencia. López-Cabarcos et al. (2017) plantearon que el desbalance esfuerzo-recompensa, independientemente de la intensidad de la VL (puntaje NAQ), puede afectar la salud mental, particularmente en mujeres.

El hecho de que el efecto del desbalance esfuerzo-recompensa en el distrés esté en parte mediado por la VL se relaciona con que esta última, independientemente del desbalance esfuerzo- recompensa, tiene efectos negativos en la salud mental de los trabajadores/as, lo que se encuentra ampliamente reportado en la literatura (Baby et al., 2014; Dement et al., 2014; Einarsen \& Nielsen, 2015; Itzhaki et al., 2015; Magnavita, 2014; Pien et al., 2015; Rodwell \& Demir, 2012; Zhao et al., 2018).

En este estudio se encontró evidencia respecto a que la exposición a liderazgo tiránico se relaciona directamente con el nivel de exposición a VL y a que el efecto de la exposición a liderazgo tiránico en el distrés se encuentra completamente mediado por la exposición a VL. La relación positiva entre exposición a liderazgo tiránico y el nivel de exposición a VL coincide con estudios previos, como el de Hoel et al. (2010), en el que se encontró que este tipo de liderazgo es predictor del bullying observado. Además, coincide con lo reportado por 
González (2019) en una muestra de trabajadores/as chilenos/as, en la que la exposición a liderazgo tiránico se asocia con la exposición a VL. Dicha asociación se produce porque jefes/as que adscriben a los objetivos organizacionales, aun a expensas del bienestar laboral de los/as trabajadores/as, suelen usar las amenazas o la intimidación para que los/as trabajadores/as alcancen las metas.

A su vez, la relación entre liderazgo tiránico y distrés se condice con investigación previa, como la de Nielsen et al. (2005), quienes, en un estudio con trabajadores noruegos víctimas de intimidación, encontraron que los líderes tiránicos constituyen un factor de estrés severo, o el de Stordeur et al. (2001), quienes reportan que el estilo de liderazgo influye en el agotamiento emocional.

Contrariamente a lo hipotetizado en este estudio, no se encontró un efecto directo del liderazgo tiránico en el distrés, sino que solamente un efecto indirecto. Es decir, el liderazgo tiránico aumentaría el distrés solamente en cuanto se asocia con mayor probabilidad de estar expuesto a la VL. La ausencia de un efecto directo de liderazgo tiránico se puede deber a tres motivos. Primero, se puede relacionar con la población del estudio. El sector salud es un sector extremadamente jerarquizado (Ansoleaga Moreno et al., 2012) y, en consecuencia, quienes se desempeñan en salud se han habituado a líderes que establecen relaciones verticales y autoritarias, con alto diferencial de poder entre jefes y subalternos, que es el estilo característico de las jefaturas en Chile (Ramos, 2014, citado en Díaz Berr et al., 2017). Es decir, están acostumbrados a líderes que ejercen presión para lograr eficiencia e impiden la autonomía de los/as funcionarios/as (Rodríguez \& Gómez, 2009, citado en Díaz Berr et. al., 2017). Sin embargo, cuando ello se une a la percepción de VL, produce distrés en los trabajadores/as. Segundo, porque el concepto de liderazgo tiránico no incluye las intenciones o motivos de parte del líder (Einarsen et al., 2010) y el no percibir la intención de dañar, puede ser un elemento protector para el aumento de distrés. Sin embargo, si el liderazgo tiránico se traduce en la exposición a VL, los trabajadores/as observan una clara intención de daño (Franco, 2015), aumentando el nivel de distrés. En tercer lugar, porque el estar expuesto a liderazgo tiránico no excluye que se esté expuesto simultáneamente a formas constructivas de liderazgo (Einarsen et al., 2007, 2010). Así, este hallazgo contrario a la literatura previa que vincula el liderazgo (Stordeur et al., 2001) y particularmente el liderazgo tiránico (Einarsen et al., 2010; Nielsen et al., 2005) con problemas de salud mental, se puede comprender porque los trabajadores/as pueden haber estado simultáneamente expuestos a liderazgos constructivos que los protegen del distrés.

En esta muestra las covariables analizadas se comportan y distribuyen de manera desigual entre hombres y mujeres y entre estamentos, confirmando, de este modo, la reproducción en el espacio laboral de las desigualdades sociales y, con ello, la relevancia de considerar la importancia de la asimetría de poder al estudiar la violencia al interior de las organizaciones (Díaz Berr et al., 2017; Escartín et al., 2012; Krieger, 2001; Salin, 2003).

Los hallazgos de este estudio hacen pensar en la necesidad de incorporar la VL en los protocolos de vigilancia de riesgos psicosociales, puesto que parte de los efectos de dichos riesgos en el distrés de los/as trabajadores/as se debería a la presencia simultánea de VL. Además, se considera necesario implementar medidas preventivas de VL que pongan énfasis en aspectos de la organización del trabajo que se encuentran asociados con los fenómenos ISOSTRAIN, desbalance esfuerzo-recompensa y liderazgo tiránico y medidas reparadoras, debido a la evidencia de consecuencias en el distrés de los/as trabajadores/as. En materia de medidas preventivas, es posible incorporar políticas de tolerancia cero contra la violencia en las organizaciones. También se ha considerado el concepto de infraestructura ética en las organizaciones que define los comportamientos aceptables, señala las posibles consecuencias si se violan las normas de conductas y actúa como guía para responder eficazmente a los comportamientos no éticos o violentos (Einarsen et al., 2019).

Entre las limitaciones de este estudio, se encuentra el carácter transversal del mismo, que impide establecer relaciones causa-efecto, la muestra no aleatoria, que impide extrapolar los hallazgos a otras poblaciones, y la posible deseabilidad social en las respuestas respecto a VL y sintomatología de problemas de salud mental.

Para lograr establecer causalidad empírica y extrapolación de los hallazgos a la población de trabajadores/as sanitarios/as de Chile, se sugiere realizar futuros estudios con diseños longitudinales y con muestras aleatorias que incluyan trabajadores/as que se desempeñen en centros asistenciales de salud con diversos niveles de complejidad. Además, se sugiere realizar estudios que permitan realizar análisis clínicos de los resultados. 


\section{Referencias}

Aasland, M. S., Skogstad, A., Notelaers, G., Nielsen, M. B. \& Einarsen, S. (2010). The prevalence of destructive leadership behaviour. British Journal of Management, 21(2), 438-452. https://doi.org/10.1111/j.1467-8551.2009.00672.x

Al-Azzam, M., Tawalbeh, L., Sulaiman, M., Al-Sagarat, A. Y. \& Harb, E. (2017). Prevalence and risk factors of workplace violence toward mental health staff departments in Jordanian hospitals. Issues in Mental Health Nursing, 38(5), $435-442$. https://doi.org/10.1080/01612840.2016.1268225

Amable, M. (2006). La precariedad laboral y su impacto sobre la salud. Un estudio en trabajadores asalariados en España [Memoria de doctorado, Universitat Pompeu Fabra]. Tesis Doctorals en Xarxa. https://www.tdx.cat/handle/10803/7116

Ansoleaga, E. (2015). Indicadores de salud mental asociados a riesgo psicosocial laboral en un hospital público. Revista Médica de Chile, 143(1), 47-55. https://doi.org/10.4067/S0034-98872015000100006

Ansoleaga, E., Ahumada, M. \& González-Santa Cruz, A. (2019). Association of workplace bullying and workplace vulnerability in the psychological distress of Chilean workers. International Journal of Environment Research and Public Health, 16(20), Artículo 4039. https://doi.org/10.3390/ijerph16204039

Ansoleaga, E., Díaz, X., Mauro, A. \& Toro, J. P. (2020). Prevalencia, formas de manifestación y factores facilitadores de la violencia laboral, y sus efectos en la salud mental en población asalariada en Chile atendiendo desigualdades sociales y de género [Proyecto FONDECYT Regular $\mathrm{N}^{\mathrm{o}}$ 1170239]. Universidad Diego Portales. https://pepet.udp.cl/wp-content/uploads/2017/10/Abstract-fondecyt-2017.pdf

Ansoleaga, E., Montaño, R. \& Vézina, M. (2013). Validation of two complementary instruments for measuring work stress in Chilean workers. Scandinavian Journal of Organizational Psychology, https://www.researchgate.net/publication/259309577_Validation_of_two_complementary_instruments_for_measuring_work_stres s in Chilean workers

Ansoleaga Moreno, E., Artaza Barrios, O. \& Suárez Jiménez, J. M. (Eds.). (2012). Personas que cuidan personas: dimensión humana y trabajo en salud. Organización Mundial de la Salud, Organización Panamericana de la Salud. https://iris.paho.org/handle/10665.2/52631

Ansoleaga Moreno, M. E. (2013). Sintomatología depresiva y consumo riesgoso de alcohol en trabajadores expuestos a factores psicosociales laborales adversos: un asunto pendiente en salud pública [Tesis de doctorado, Universidad de Chile]. Repositorio Académico de la Universidad de Chile. http://repositorio.uchile.cl/handle/2250/116472

Araujo, K. (2014). La desmesura y los sujetos: el trabajo en el caso de Chile. En A. Stecher \& L. Godoy (Eds.). Transformaciones del trabajo, subjetividad e identidades: lecturas psicosociales desde Chile y América Latina (pp. 277-298). RIL.

Ariza-Montes, A., Muniz, N. M., Montero-Simó, M. J. \& Araque-Padilla, R. A. (2013). Workplace bullying among health care workers. International Journal of Environmental Research and Public Health, 10(8), 3121-3139. https://doi.org/10.3390/ijerph10083121

Arnetz, J., Hamblin, L., Sudan, S. \& Arnetz, B. (2018). Organizational determinants of workplace violence against hospital workers. Journal of Occupational and Environmental Medicine, 60(8), 693-699. https://doi.org/10.1097/JOM.0000000000001345

Aronsson, V., Toivanen, S., Leineweber, C. \& Nyberg, A. (2019). Can a poor psychosocial work environment and insufficient organizational resources explain the higher risk of ill-health and sickness absence in human service occupations? Evidence from a Swedish national cohort. Scandinavian Journal of Public Health, 47(3), 310-317. https://doi.org/10.1177/1403494818812638

Artaza, O. (2012). La reforma a la salud en Chile. En E. Ansoleaga Moreno, O. Artaza Barrios \& J. M. Suárez Jiménez (Eds.), Personas que cuidan personas: dimensión humana y trabajo en salud (pp. 52-69). Organización Mundial de la Salud, Organización Panamericana de la Salud. https://iris.paho.org/handle/10665.2/52631

Ashforth, B. (1994). Petty tyranny in organizations. Human Relations, 47(7), 755-778. https://doi.org/10.1177/001872679404700701

Attell, B. K., Kummerow Brown, K. \& Treiber, L. A. (2017). Workplace bullying, perceived job stressors, and psychological distress: Gender and race differences in the stress process. Social Science Research, 65, 210-221. https://doi.org/10.1016/j.ssresearch.2017.02.001

Baby, M., Glue, P. \& Carlyle, D. (2014). 'Violence is not part of our Job': A thematic analysis of psychiatric mental health nurses' experiences of patient assaults from a New Zealand perspective. Issues in Mental Health Nursing, 35(9), 647-655. https://doi.org/10.3109/01612840.2014.892552

Balducci, C., Fraccaroli, F. \& Schaufeli, W. B. (2011). Workplace bullying and its relation with work characteristics, personality, and post-traumatic stress symptoms: An integrated model. Anxiety, Stress \& Coping, 24(5), 499-513. https://doi.org/10.1080/10615806.2011.555533

Candia, M., Pérez Franco, J. M. \& González, D. (2016). Manual del método del Cuestionario SUSESO/ISTAS21: versiones completa y breve. Gobierno de Chile, Superintendencia de Seguridad Social, Intendencia de Seguridad y Salud en el Trabajo, Unidad de Medicina del Trabajo. https://www.suseso.cl/613/articles-481095_archivo_03.pdf

Carretero Domínguez, N., Gil-Monte, P. R. \& Luciano Devis, J. V. (2011). Antecedentes y consecuencias del acoso psicológico en el trabajo. Psicothema, 23(4), 617-623. http://www.psicothema.com/pdf/3931.pdf

Casas Becerra, L. (2016). The effectiveness of sexual harassment law in Chile: From theory to practice [Tesis de doctorado, University of Ottawa]. uO Research. https://ruor.uottawa.ca/bitstream/10393/34670/1/Casas_Lidia_2016 thesis.pdf

Cavanaugh, C., Campbell, J. \& Messing, J. T. (2014). A longitudinal study of the impact of cumulative violence victimization on comorbid posttraumatic stress and depression among female nurses and nursing personnel. Workplace Health \& Safety, 62(6), $224-232$. https://doi.org/10.1177/216507991406200602

Centro de Estudios de la Mujer \& Universidad de Ottawa. (2011). Encuesta Araucaria. Centro de Estudios de la Mujer. https://cem.cl/proyecto-12/

Cheng, W. -J. \& Cheng, Y. (2017). Minor mental disorders in Taiwanese healthcare workers and the associations with psychosocial work conditions. Journal of the Formosan Medical Association, 116(4), 300-305. https://doi.org/10.1016/j.jfma.2016.05.004

Dement, J. M., Lipscomb, H. J., Schoenfisch, A. L. \& Pompeii, L. A. (2014). Impact of hospital type II violent events: Use of psychotropic drugs and mental health services. American Journal of Industrial Medicine, 57(6), 627-639. https://doi.org/10.1002/ajim.22306

Demir, D. \& Rodwell, J. (2012). Psychosocial antecedents and consequences of workplace aggression for hospital nurses. Journal of Nursing Scholarship, 44(4), 376-384. https://doi.org/10.1111/j.1547-5069.2012.01472.x

Departamento de Salud Ocupacional. (2013). Protocolo de vigilancia de riesgos psicosociales en el trabajo. Gobierno de Chile, Ministerio de Salud, Subsecretaría de Salud Pública, División de Políticas Públicas Saludables y Promoción. https://www.minsal.cl/portal/url/item/e039772356757886e040010165014a72.pdf

De Witte, H. (1999). Job insecurity and psychological well-being: Review of the literature and exploration of some unresolved issues. European Journal of Work and Organizational Psychology, 8(2), 155-177. https://doi.org/10.1080/135943299398302 
Díaz Berr, X., Mauro Cardarelli, A., Ansoleaga Moreno, E. \& Toro Cifuentes, J. P. (2017). Violencia de género en el trabajo en Chile. Un campo de estudio ignorado. Ciencia \& Trabajo, 19(58), 42-48. https://doi.org/10.4067/S0718-24492017000100042

Di Martino, V. (2002). Workplace violence in the health sector. Country case studies: Brazil, Bulgaria, Lebanon, Portugal, South Africa, Thailand and an additional Australian study. Synthesis report. International Labor Organization https://www.who.int/violence_injury_prevention/injury/en/WVsynthesisreport.pdf

Di Martino, V., Hoel, H. \& Cooper, C. L. (2003). Preventing violence and harassment in the workplace. European Foundation for the improvement of living and working conditions. European Foundation for the Improvement of Living and Working Conditions. http://www.eurofound.europa.eu/sites/default/files/ef files/pubdocs/2002/109/en/1/ef02109en.pdf

Einarsen, K., Salin, D., Einarsen. S. V., Skogstad, A. \& Mykletun, R. J. (2019). Antecedents of ethical infrastructures against workplace bullying: The role of organizational size, perceived financial resources and level of high-quality HRM practices. Personnel Review, 48(3), 672-690. https://doi.org/10.1108/PR-10-2017-0303

Einarsen, S. (2000). Harassment and bullying at work: A review of the Scandinavian approach. Aggression and Violent Behavior, 5(4), 379-401. https://doi.org/10.1016/S1359-1789(98)00043-3

Einarsen, S., Aasland, M. S. \& Skogstad, A. (2007). Destructive leadership behaviour: A definition and conceptual model. The Leadership Quarterly, 18(3), 207-216. https://doi.org/10.1016/j.leaqua.2007.03.002

Einarsen, S. \& Nielsen, M. B. (2015). Workplace bullying as an antecedent of mental health problems: A five-year prospective and representative study. International Archives of Occupational and Environmental Health, 88(2), 131-142. https://doi.org/10.1007/s00420-014-0944-7

Einarsen, S., Skogstad, A. \& Aasland, M. S. (2010). The nature, prevalence, and outcomes of destructive leadership: A behavioral and conglomerate approach. En B. Schyns \& T. Hansbrough (Eds.), When leadership goes wrong: Destructive leadership, mistakes, and ethical failures (pp. 145-174). Information Age.

Escartín, J., Sora, B., Rodríguez-Muñoz, A. \& Rodríguez-Carballeira, A. (2012). Adaptación y validación de la versión española de la Escala de Conductas Negativas en el Trabajo Realizadas por Acosadores: NAQ-Perpetrators. Revista de Psicología del Trabajo y de las Organizaciones, 28(3), 157-170. https://doi.org/10.5093/tr2012a13

Fardella, C., Sisto, V., Morales, K., Rivera, G. \& Soto, R. (2016). Identidades laborales y ética del trabajo público en tiempos de rendición de cuentas. Psykhe, 25(2), Artículo 7. https://doi.org/10.7764/psykhe.25.2.789

Feijó, F., Fassa, A. \& Pearce, N. (2019). What psychosocial factors at work are associated to workplace bullying? A study with judiciary Brazilian civil servants from Southern Brazil. Occupational and Environmental Medicine, 76(Suppl 1), Artículo A14. https://doi.org/10.1136/OEM-2019-EPI.37

Fischer, F. M., Silva-Costa, A., Griep, R. H., Smolensky, M. H., Bohle, P. \& Rotenberg, L. (2019). Working time society consensus statements: Psychosocial stressors relevant to the health and wellbeing of night and shift workers. Industrial Health, 57(2), 175183. https://doi.org/10.2486/indhealth.SW-3

Fontes, K. B., Alarcão, A. C. J., Santana, R. G., Pelloso, S. M. \& de Barros Carvalho, M. D.n (2019). Relationship between leadership, bullying in the workplace and turnover intention among nurses. Journal of Nursing Management, 27(3), 535-542. https://doi.org/10.1111/jonm.12708

Francioli, L., Conway, P. M., Hansen, A. M., Holten, A. -L., Grynderup, M. B., Persson, R., Mikkelsen, E. G., Costa, G. \& Høgh, A. (2018). Quality of leadership and workplace bullying: The mediating role of social community at work in a two-year follow-up study. Journal of Business Ethics, 147(4), 889-899. https://doi.org/10.1007/s10551-015-2996-3

Franco, S. (2015). Violencia psicológica en el trabajo. Caracterización e impacto en los trabajadores y las organizaciones [Tesis de doctorado, Universidad de Deusto]. DKH Thesis. https://dkh.deusto.es/comunidad/thesis/recurso/violencia-psicologica-en-eltrabajo/dca26252-99f2-45ab-90ec-f746f7376f21

Glambek, M., Matthiesen, S. B., Hetland, J. \& Einarsen, S. (2014). Workplace bullying as an antecedent to job insecurity and intention to leave: A 6-month prospective study. Human Resource Management Journal, 24(3), 255-268. https://doi.org/10.1111/1748-8583.12035

Gong, Y., Han, T., Chen, W., Dib, H. H., Yang, G., Zhuang, R., Cheng, Y., Tong, X., Yin, X. \& Lu, Z. (2014). Prevalence of anxiety and depressive symptoms and related risk factors among physicians in China: A cross-sectional study. PLoS One, 9(7), Artículo e103242. https://doi.org/10.1371/journal.pone.0103242

González, A. (2019). Propiedades psicométricas de escala de liderazgo destructivo y cuestionario de conductas negativas-revisado en una muestra multiocupacional de trabajadores dependientes chilenos de Gran Santiago, Gran Valparaíso y Gran Concepción [Tesis de magíster no publicada]. Universidad Diego Portales.

Greenhalgh, L. \& Rosenblatt, Z. (1984). Job insecurity: Toward conceptual clarity. The Academy of Management Review, 9(3), 438-448. https://doi.org/10.5465/\%20amr.1984.4279673

Han, K. -M., Chang, J., Won, E., Lee, M. -S. \& Ham, B. -J. (2017). Precarious employment associated with depressive symptoms and suicidal ideation in adult wage workers. Journal of Affective Disorders, 218, 201-209. https://doi.org/10.1016/j.jad.2017.04.049

Hoel, H., Glaso, L., Hetland, J., Cooper, C. L. \& Einarsen, S. (2010). Leadership styles as predictors of self-reported and observed workplace bullying. British Journal of Management, 21(2), 453-468. https://doi.org/10.1111/j.1467-8551.2009.00664.x

Hopenhayn, M. (2001). Repensar el trabajo: historia, profusión y perspectivas de un concepto. Editorial Norma.

Itzhaki, M., Peles-Bortz, A., Kostisky, H., Barnoy, D., Filshtinsky, V. \& Bluvstein, I. (2015). Exposure of mental health nurses to violence associated with job stress, life satisfaction, staff resilience, and post-traumatic growth. International Journal of Mental Health Nursing, 24(5), 403-412. https://doi.org/10.1111/inm.12151

Julià, M., Vanroelen, C., Bosmans, K., Van Aerden, K. \& Benach, J. (2017). Precarious employment and quality of employment in relation to health and well-being in Europe. International Journal of Health Services, 47(3), 389-409. https://doi.org/10.1177/0020731417707491

Karasek, R., Brisson, C., Kawakami, N., Houtman, I., Bongers, P. \& Amick, B. (1998). The Job Content Questionnaire (JCQ): An instrument for internationally comparative assessments of psychosocial job characteristics. Journal of Occupational Health Psychology, 3(4), 322-355. https://doi.org/10.1037/1076-8998.3.4.322

Karasek, R. \& Theorell, T. (1990). Healthy work: Stress, productivity, and the reconstruction of working life. Basic Books.

Kessler, R. C., Andrews, G., Colpe, L. J., Hiripi, E., Mrocsek, D. K., Normand, S. -L. T., Walters, E. E. \& Zaslavsky, A. M. (2002). Short screening scales to monitor population prevalences and trends in non-specific psychological distress. Psychological Medicine, 32(6), 959-976. https://doi.org/10.1017/s0033291702006074

Keuskamp, D., Ziersch, A. M., Baum, F. E. \& LaMontagne, A. D. (2012). Workplace bullying a risk for permanent employees. Australian and New Zealand Journal of Public Health, 36(2), 116-119. https://doi.org/10.1111/j.1753-6405.2011.00780.x 
Krieger, N. (2001). Theories for social epidemiology in the 21st century: An ecosocial perspective. International Journal of Epidemiology, 30(4), 668-677. https://doi.org/10.1093/ije/30.4.668

Llosa, J. A., Menéndez-Espina, S., Agulló-Tomás, E. \& Rodríguez-Suárez, J. (2018). Job insecurity and mental health: A meta-analytical review of the consequences of precarious work in clinical disorders. Anales de Psicología, 34(2), 211-223. https://doi.org/10.6018/analesps.34.2.281651

Lopera, J. D. (2014). El concepto de salud mental en algunos instrumentos de políticas públicas de la Organización Mundial de la Salud. Revista Facultad Nacional de Salud Pública, 32(Suplemento 1), S11-S20. https://revistas.udea.edu.co/index.php/fnsp/article/view/19792/18466

López-Cabarcos, M. Á., Vázquez-Rodríguez, P. \& Gieure, C. (2017). Gender and age differences in the psychosocial risk factors of workplace bullying. Psychology \& Marketing, 34(11), 1023-1030. https://doi.org/10.1002/mar.21041

Magnavita, N. (2014). Workplace violence and occupational stress in healthcare workers: A chicken-and-egg situation: Results of a 6year follow-up study. Journal of Nursing Scholarship, 46(5), 366-376. https://doi.org/10.1111/jnu.12088

Malik, O. F., Schat, A. C. H., Shahzad, A., Raziq, M. M. \& Faiz, R. (2018). Workplace psychological aggression, job stress, and vigor: A test of longitudinal effects. Journal of Interpersonal Violence, 36(5-6). https://doi.org/10.1177/0886260518770650

Mnookin, S. (2016). Out of the shadows: Making mental health a global development priority. World Bank Group \& World Health Organization. http://documents.worldbank.org/curated/pt/270131468187759113/pdf/105052-WP-PUBLIC-wb-background-paper.pdf

Moncada, S., Llorens, C. \& Kristensen, T. S. (2002). Método ISTAS21 (CoPsoQ): manual para la evaluación de riesgos psicosociales en el trabajo. Instituto Sindical de Trabajo Ambiente y Salud. http://istas.net/descargas/m_metodo_istas21.pdf

Moscone, F., Tosetti, E. \& Vittadini, G. (2016). The impact of precarious employment on mental health: The case of Italy. Social Science \& Medicine, 158, 86-95. https://doi.org/10.1016/j.socscimed.2016.03.008

Niedhammer, I., Lesuffleur, T., Coutrot, T. \& Chastang, J. -F. (2016). Contribution of working conditions to occupational inequalities in depressive symptoms: Results from the national French SUMER survey. International Archives of Occupational and Environmental Health, 89(6), 1025-1037. https://doi.org/10.1007/s00420-016-1142-6

Nielsen, M. B., Matthiesen, S. B. \& Einarsen, S. (2005). Ledelse og personkonflikter: Symptomer på posttraumatisk stress blant ofre for mobbing fra ledere. [Liderazgo y conflictos interpersonales: Síntomas de estrés postraumático en víctimas de acoso por parte de líderes]. Nordisk Psykologi, 57, 391-415. https://doi.org/10.1080/00291463.2005.10637381

Nielsen, M. B., Tvedt, S. D. \& Matthiesen, S. B. (2013). Prevalence and occupational predictors of psychological distress in the offshore petroleum industry: A prospective study. International Archives of Occupational and Environmental Health, 86(8), 875-885. https://doi.org/10.1007/s00420-012-0825-x

Notelaers, G., De Witte, H. \& Einarsen, S. (2010). A job characteristics approach to explain workplace bullying. European Journal of Work and Organizational Psychology, 19(4), 487-504. https://doi.org/10.1080/13594320903007620

Notelaers, G. \& Einarsen, S. (2013). The world turns at 33 and 45: Defining simple cutoff scores for the Negative Acts Questionnaire-Revised in a representative sample. European Journal of Work and Organizational Psychology, 22(6), 670-682. https://doi.org/10.1080/1359432X.2012.690558

Notelaers, G., Vermunt, J. K., Baillien, E., Einarsen, S. \& De Witte, H. (2011). Exploring risk groups workplace bullying with categorical data. Industrial Health, 49(1), 73-88. https://doi.org/10.2486/indhealth.MS1155

Obeidat, R. F., Qan'ir, Y. \& Turaani, H. (2018). The relationship between perceived competence and perceived workplace bullying among registered nurses: A cross sectional survey. International Journal of Nursing Studies, 88, 71-78. https://doi.org/10.1016/j.ijnurstu.2018.08.012

Olsen, E., Bjaalid, G. \& Mikkelsen, A. (2017). Work climate and the mediating role of workplace bullying related to job performance, job satisfaction, and work ability: A study among hospital nurses. Journal of Advanced Nursing, 73(11), $2709-2719$. https://doi.org/10.1111/jan.13337

Organización Internacional del Trabajo, Consejo Internacional de Enfermeras, Organización Mundial de la Salud \& Internacional de Servicios Públicos. (2002). Directrices marco para afrontar la violencia laboral en el sector de salud. http://apps.who.int/iris/bitstream/10665/44072/1/9223134463_spa.pdf?ua=1

Palma-Contreras, A. \& Ansoleaga, E. (2020). Asociaciones entre factores de riesgos psicosociales, dimensiones organizacionales y problemas de salud mental, relacionados con la violencia laboral, en trabajadores de tres hospitales chilenos de alta complejidad. Cadernos de Saúde Pública, 36(3), Artículo e00084219. https://doi.org/10.1590/0102-311X00084219

Park, J. H. \& Ono, M. (2017). Effects of workplace bullying on work engagement and health: The mediating role of job insecurity. The International Journal of Human Resource Management, 28(22), 3202-3225. https://doi.org/10.1080/09585192.2016.1155164

Pien, L. -C., Chen, D. -R., Chen, C. -J, Liang, K. -M. \& Cheng, Y. (2015). Associations of neighborhood-level workplace violence with workers' mental distress problems: A multilevel analysis of Taiwanese employees. Journal of Occupational Health, 57(6), 555-564. https://doi.org/10.1539/joh.15-0132-OA

Radey, M. \& Wilke, D. J. (2018). Client-perpetrated violence among frontline child welfare workers. Journal of Interpersonal Violence. Anticipo en línea de la publicación. https://doi.org/10.1177/0886260518812792

Reknes, I., Notelaers, G., Magerøy, N., Pallesen, S., Bjorvatn, B., Moen, B. E. \& Einarsen, S. (2017). Aggression from patients or next of kin and exposure to bullying behaviors: A conglomerate experience? Nursing Research and Practice, 2017 , Article 1502854. https://doi.org/10.1155/2017/1502854

Rosseel, Y. (2012). lavaan: An R package for structural equation modeling. Journal of Statistical Software, 48, Artículo 2. https://doi.org/10.18637/jss.v048.i02

Rodwell, J. \& Demir, D. (2012). Psychological consequences of bullying for hospital and aged care nurses. International Nursing Review, 59(4), 539-546. https://doi.org/10.1111/j.1466-7657.2012.01018.x

Russo, M., Lucifora, C., Pucciarelli, F. \& Piccoli, B. (2019). Work hazards and workers' mental health: An investigation based on the fifth European Working Conditions Survey. La Medicina del Lavoro, 110(2), 115-129. https://doi.org/10.23749/mdl.v110i2.7640

Salas, M. L., Quezada, S., Basagoitia, A., Fernandez, T., Herrera, R., Parra, M., Moraga Muñoz, D., Weigl, M. \& Radon, K. (2015). Working conditions, workplace violence, and psychological distress in Andean miners: A cross-sectional study across three countries. Annals of Global Health, 81(4), 465-474. https://doi.org/10.1016/j.aogh.2015.06.002

Salin, D. (2003). Ways of explaining workplace bullying: A review of enabling, motivating and precipitating structures and processes in the work environment. Human Relations, 56(10), 1213-1232. https://doi.org/10.1177/00187267035610003

Seun-Fadipe, C. T., Akinsulore, A. A. \& Oginni, O. A. (2019). Workplace violence and risk for psychiatric morbidity among health workers in a tertiary health care setting in Nigeria: Prevalence and correlates. Psychiatry Research, 272, 730-736. https://doi.org/10.1016/j.psychres.2018.12.177 
Siegrist, J., Wege, N., Pühlhofer, F. \& Waherendorf, M. (2009). A short generic measure of work stress in the era of globalization: Effortreward imbalance. International Archives of Occupational and Environmental Health, 82(8), Article 1005. https://doi.org/10.1007/s00420-008-0384-3

Sisto, V. (2012). Identidades desafiadas: individualización, managerialismo y trabajo docente en el Chile actual. Psykhe, 21(2), 35-46. https://doi.org/10.7764/psykhe.21.2.542

Spagnoli, P. \& Balducci, C. (2017). Do high workload and job insecurity predict workplace bullying after organizational change? International Journal of Workplace Health Management, 10(1), 2-12. https://doi.org/10.1108/IJWHM-05-2016-0038

Spence Laschinger, H. K., Wong, C. A. \& Grau, A. L. (2012). The influence of authentic leadership on newly graduated nurses' experiences of workplace bullying, burnout and retention outcomes: A cross-sectional study. International Journal of Nursing Studies, 49(10), 1266-1276. https://doi.org/10.1016/j.ijnurstu.2012.05.012

Sperduti, A., Hindle, D., Shessel, A., Pidgeon, B., Akmal, H., Chaulk, G., Di Tomasso, A., Divanbeigi, L. \& Rosewall, T. (2018). Treating too lightly? Radiation therapists' experiences of workplace violence when providing care to cancer patients and their families. Journal of Medical Imaging and Radiation Sciences, 49(1), 56-61. https://doi.org/10.1016/j.jmir.2017.11.001

Stecher Guzmán, A. (2012). Procesos de construcción de identidades laborales de trabajadores de la industria del retail en Santiago de Chile. Contribuciones al estudio de las subjetividades laborales en el nuevo capitalismo [Tesis de doctorado, Universitat Autònoma de Barcelona]. TESEO. https://www.educacion.gob.es/teseo/imprimirFicheroTesis.do?idFichero=1ck6YrnwHJY\%3D

Stordeur, S., D'Hoore, W. \& Vandenberghe, C. (2001). Leadership, organizational stress, and emotional exhaustion among hospital nursing staff. Journal of Advanced Nursing, 35(4), 533-542. https://doi.org/10.1046/j.1365-2648.2001.01885.x

Tonso, M. A., Prematunga, R. K., Norris, S. J., Williams, L., Sands, N. \& Elsom, S. J. (2016). Workplace violence in mental health: A Victorian mental health workforce survey. International Journal of Mental Health Nursing, $25(5), 444-451$. https://doi.org/10.1111/inm.12232

Toro, J. P. \& Gómez-Rubio, C. (2016). Factores facilitadores de la violencia laboral: una revisión de la evidencia científica en América Latina. Ciencia \& Trabajo, 18(56), 110-116. https://doi.org/10.4067/S0718-24492016000200006

Tsuno, K., Kawakami, N., Tsutsumi, A., Shimazu, A., Inoue, A., Odagiri, Y., Yoshikawa, T., Haratani, T., Shimomitsu, T. \& Kawachi, I. (2015). Socioeconomic determinants of bullying in the workplace: A national representative sample in Japan. PLoS One, 10(3), Artículo e0119435. https://doi.org/10.1371/journal.pone.0119435

Tsutsumi, A., Izutsu, T., Ito, A. \& Hashimoto, J. (2015). Mental health, well-being and disability: A new global priority. Key United Nations resolutions and documents. United Nations University, United Nations, World Bank Group \& The University of Tokyo. http://i.unu.edu/media/iigh.unu.edu/news/3735/UN-MHWD-Book-2015.pdf

van der Velden, P. G., Bosmans, M. W. G. \& van der Meulen, E. (2016). Predictors of workplace violence among ambulance personnel: A longitudinal study. Nursing Open, 3(2), 90-98. https://doi.org/10.1002/nop2.38

Wei, C. -Y., Chiou, S. -T., Chien, L. -Y. \& Huang, N. (2016). Workplace violence against nurses - Prevalence and association with hospital organizational characteristics and health-promotion efforts: Cross-sectional study. International Journal of Nursing Studies, 56, 63-70. https://doi.org/10.1016/j.ijnurstu.2015.12.012

World Health Organization. (2000). The world health report 2000. Health systems: Improving performance. http://www.who.int/whr/2000/en/whr00_en.pdf

Yada, H., Abe, H., Omori, H., Matsuo, H., Masaki, O., Ishida, Y. \& Katoh, T. (2014). Differences in job stress experienced by female and male Japanese psychiatric nurses. International Journal of Mental Health Nursing, 23(5), 468-476. https://doi.org/10.1111/inm.12080

Zafar, W., Khan, U. R., Siddiqui, S. A., Jamali, S. \& Razzak, J. A. (2016). Workplace violence and self-reported psychological health: Coping with post-traumatic stress, mental distress, and burnout among physicians working in the emergency departments compared to other specialties in Pakistan. The Journal of Emergency Medicine, 50(1), 167-177.e1. https://doi.org/10.1016/j.jemermed.2015.02.049

Zhao, S., Xie, F., Wang, J., Shi, Y., Zhang, S., Han, X., Sun, Z., Shi, L., Li, Z., Mu, H., Liu, X., Liu, W., Gao, L., Sun, T. \& Fan, L. (2018). Prevalence of workplace violence against Chinese nurses and its association with mental health: A cross-sectional survey. Archives of Psychiatric Nursing, 32(2), 242-247. https://doi.org/10.1016/j.apnu.2017.11.009

Fecha de recepción: Diciembre de 2019.

Fecha de aceptación: Abril de 2021. 